\title{
Interference Management Strategy for Deploying Energy Efficient Femtocells Network
}

\author{
Aawatif Hayar*, Bassem Zayen ${ }^{\dagger}$ and Sofia Bouferda ${ }^{\ddagger}$ \\ *GREENTIC-ENSEM, Hassan II University, Casablanca, Morocco \\ ${ }^{\dagger}$ EURECOM, Mobile Communications Department, Sophia Antipolis, France \\ ${ }^{\ddagger}$ GREENTIC-ESTC, Hassan II University, Casablanca, Morocco
}

\begin{abstract}
In this paper, we propose a new interference management scheme for femtocells systems based on the cooperation and inter-handover techniques between competing cells in the context of cognitive radio systems. The original idea presented in this work consists of initiating a cooperation protocol between femtocells through inter-handover algorithm based on channel gain diversity. Users belong a given femtocell are allowed to connect to a neighboring cell if their channel gains to this cell are better than what they have with their own femtocell. We have shown in this work, using a game theory based resource allocation scheme, that we can achieve better performance in terms of capacity and power consumption using our approach. The results have shown also that a simple win-win cooperation protocols can be implemented to improve the energy consumption and performance of new cellular systems such as femtocells or even for classical cellular systems if a minimum cooperation schemes are allowed.
\end{abstract}

Keywords-Cognitive Radio, Interference Management, Energy Efficient, Game Theory, Femtocells Network.

\section{INTRODUCTION}

In contrast to wired devices, the lack of a continuous power supply, poses tight limits in the overall power consumption [1]. The limited battery lifetime has always been bottleneck when it comes to the development of improved portable electronic products. In addition, constraints in the size and weight of mobile phones prohibit the use of heavy and large battery packs as power sources [2] [3]. Although, battery technology has been improved over the years, it definitely has not kept up with the advances in other technological fields or the energy demands of wireless platforms. Apart from the short battery life span, another factor that makes power consumption a critical parameter for personal portable devices is the heat dissipation. The latter needs to remain at low levels, otherwise the system's temperature will increase, making the device too hot to be handled. Therefore, minimizing the power consumption of wireless platforms becomes a great challenge, for the entire electronic industry, at all system levels [2] [3].

Also, in recent years, there has been increasing public concern about the health implication of wireless systems electromagnetic radiation. Many researches have been done to give a complete picture of health risks that are caused by the use of mobile phones also various public organizations in the world have been established safety guidelines like a limit of absorption rate (SAR) of mobile that is stated by International Commission on Non-Ionization Radiation Protection (ICNIRP) [4].
To cope with the issues mentioned above, we propose to consider the deployment of small cells termed in the femtocells to reduce the transmit power and optimize energy consumption. Indeed, femtocells is interesting and radically different network design concept which could provide a costand energy efficient solution to cope with the forecasted traffic growth. Briefly, Home NodeBs (HNB) networks are built on a very dense deployment of low cost, low-power base stations (BSs) that are substantially smaller than traditional macro cell equipment.

In this paper we consider a scenario with cognitive femtocells operating in the same band which arises the problem of interference management to allow co-existence between differents co-located femtocells. We propose here to combine handover based cooperative protocol with cognitive radio (CR) and game theory approaches to mitigate interference and improve performance at the same time [5] [6] [7]. Indeed, cooperation is used to reduce energy consumption, improve channel condition and thus optimize system capacity. The proposed technique will be compared with classical one based on game theory tools [5].

The paper is organized as follows. In Section II we describe the channel model and introduce the proposed combined CRgame theory strategy. In Section III, the power allocation algorithm is presented. Simulation results and a comparison with a classical resource allocation method based on game theory tools are provided in Section IV, and Section V concludes the paper.

\section{ENERGY-EFFICIENCY BASED HANDOVER}

Consider the uplink of a cognitive radio network (CRN) that consists of two collocated femtocells operating at the same band in a cognitive manner as shown in Fig. 1. The objective is to minimize the interference between both cells when accessing the same spectrum. To simplify the problem, we consider that each femtocell represents a kind of primary system with respect to the other collocated femtocell acting as a secondary system and vice versa. We assume then that the primary system-like femtocell, called here the base station BS, is composed of $K$ primary users (PUs). The secondary-like femtocell is composed of $M$ secondary users (SUs) randomly distributed over the system [6]. The channel gains are i.i.d random variable. 


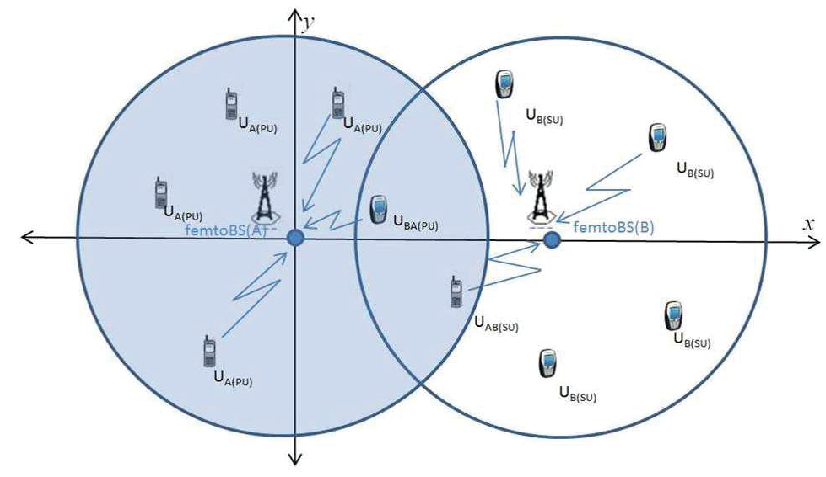

Fig. 1. Cognitive radio scenario with two collocated femtocells operating at the same band.

Throughout this paper, we will use the following notation:

- the index of SUs $m$ lies between 1 and $M$,

- $h_{l, m}$ denotes the channel gain from SU $l$ to the desired user $m$,

- the data destined from SU $m$ is transmitted with power $P_{m}$ and a maximum power $P_{\max }$,

- $h_{k, m}$ denotes the channel gain from the PU indexed by $k$ to the desired user $m$,

- the data destined from the PU $k$ is transmitted with power $P_{k}$ for $k$ between 1 and $K$.

In the coverage area of the primary system, there is an interference boundary within which no SUs can communicate in an ad-hoc manner. Thus, for the impairment experienced by the primary system to be as small as possible, a SU must be able to detect very reliably whether it is far enough away from a primary base station, i.e., in the area of possible cognitive radio operation. The expression of each PU instantaneous capacity is

$$
C_{k}=\log _{2}\left(1+\frac{P_{k}\left|h_{k, k}\right|^{2}}{\sum_{m=1}^{M} P_{m}\left|h_{m, k}\right|^{2}+\sigma^{2}}\right)
$$

where $\sigma^{2}$ is the ambient noise variance. On the other hand, by making SUs access the primary system spectrum, the $m$-th SU experiences interference from the PUs and all neighboring cochannel SU links that transmit on the same band. Accordingly, the $m$-th SU instantaneous capacity is given by:

$$
C_{m, k}=\log _{2}\left(1+\operatorname{SINR}_{m, k}\right)
$$

where

$$
\operatorname{SINR}_{m, k}=\frac{P_{m}\left|h_{m, m}\right|^{2}}{\sum_{\substack{l=1 \\ l \neq m}}^{M} P_{l}\left|h_{l, m}\right|^{2}+P_{k}\left|h_{k, m}\right|^{2}+\sigma^{2}}
$$

SUs need to recognize their communication environment and adapt the parameters of their communication scheme in order to maximize the per-user cognitive capacity, expressed as

$$
C_{\text {sum }_{k}}=\sum_{m=1}^{M} C_{m, k}
$$

while minimizing the interference to the PUs, in a distributed fashion. The sum here is made over the $M$ SUs allowed to transmit [8] [9]. Moreover, we assume that the coherence time is sufficiently large so that the channel stays constant over each scheduling period length. We also assume that SUs know the channel state information (CSI) of their own links, but have no information on the channel conditions of other SUs. No interference cancelation capability is considered. Power control is used for SUs both in an effort to preserve power and to limit interference and fading effects. The interference power is given by:

$$
\operatorname{Intf}_{m, k}=\sum_{\substack{l=1 \\ l \neq m}}^{M} P_{l}\left|h_{l, m}\right|^{2}+P_{k}\left|h_{k, m}\right|^{2}+\sigma^{2}
$$

Combining (3) and (5), we define the SINR as a function of Intf:

$$
\operatorname{SINR}_{m, k}=\frac{P_{m}\left|h_{m, m}\right|^{2}}{\operatorname{Intf}_{m, k}}
$$

and

$$
P_{m}=\frac{\operatorname{SINR}_{m, k} \operatorname{Intf}_{m, k}}{\left|h_{m, m}\right|^{2}}
$$

The protection for PU must be guaranteed in a CRN. This protection is guaranteed if the sum of all SUs transmitters' powers is not larger than the interference constraint $P_{T}$. Then, PU verifies his outage probability constraint. The interference constraint is given by:

$$
\sum_{m=1}^{M} P_{m}\left|h_{k, m}\right|^{2} \leq P_{T_{k}}
$$

and the notion of outage probability defined as the probability that the capacity of the user is below the transmitted code rate [10]. In the proposed framework, the outage probability can be expressed as [11]:

$$
P_{\text {out }_{k}} \equiv \operatorname{Prob}\left\{C_{k} \leq R_{k}\right\} \leq P_{\text {outmax }}, \forall k=1, \ldots, K
$$

where $R_{k}$ is the transmitted data rate by one primary user $k$ and $P_{\text {outmax }}$ is the maximum outage probability defined as quality of service for each PU $k$. The information about the outage failure can be carried out by a band manager that mediates between each primary and secondary users [12], or can be directly fed back from the PU to the secondary transmitters through collaboration and exchange of the CSI between the primary and secondary users as proposed in [13]. 


\section{Power Allocation Algorithm}

We derive in this section the utility function: we define a payoff function specifies the SU capacity constraint and a price function that represents the interference constraint as a function of the outage probability constraint defined for each primary user $k$ [14] [15] [16] [17]. Therefore, the price function is given by (2), and we will derive here the equation of the interference constraint $P_{T_{k}}$.

The margin of $P_{T_{k}}-\sum_{\substack{l=1 \\ l \neq m}}^{M} P_{l}\left|h_{k, l}\right|^{2}$ is the maximum interference that SU $m$ could generate to PU $k$ under the description of (8). Divide $P_{m}\left|h_{k, m}\right|^{2}$ by $P_{T_{k}}-\sum_{\substack{l=1 \\ l \neq m}}^{M} P_{l}\left|h_{k, l}\right|^{2}$, we found the interference level expression to be guaranteed for each primary user $k$ :

$$
\mathrm{L}_{\text {Intf } m, k}=\frac{P_{m}\left|h_{k, m}\right|^{2}}{P_{T_{k}}-\sum_{\substack{l=1 \\ l \neq m}}^{M} P_{l}\left|h_{k, l}\right|^{2}}
$$

which is a normalized value. As long as this ratio $\in[0,1]$, the protection for each PU is met. We compute now $P_{T_{k}}$ as a function of the outage probability.

To proceed further with the analysis and for the sake of emphasis, we introduce the PU average channel gain estimate $G$ based on the following decomposition:

$$
h_{k, k} \equiv G_{p u} * h_{k, k}^{\prime}
$$

where $h_{k, k}^{\prime}$ is the random component of channel gain and represents the normalized channel impulse response tap.

Following the equations derived in the case of one primary user and detailed in [5], the corresponding interference constraint for each PU $k$ is

$$
P_{T_{k}}=\frac{P_{k} G_{p u}^{2}}{1-2^{R_{k}}} \ln \left(1-P_{\text {out }_{k}}\right)
$$

We introduce now a utility function for which each SU adjusts its transmitted power in order to maximize it. It is composed of a payoff function expressed as the capacity $C_{m}$ of the SU, and of a price function composed of the interference level to the PU and the power consumption. Then, the utility function is expressed as follow:

$$
U_{m, k}=C_{m, k}-\left(\frac{P_{m}\left|h_{k, m}\right|^{2}}{P_{T_{k}}-\sum_{\substack{l=1 \\ l \neq m}}^{M} P_{l}\left|h_{l, m}\right|^{2}}\right)^{a_{m}}
$$

The parameter $a_{m}$ is adjustable to have a comparable values, i.e. the payoff function value and the price function value. This parameter gives the flexibility needed to adjust the SU capacity over the interference to the PU. We choose $a_{m}<0$. It could be easily obtained that the price function decreases as the ratio $\mathrm{L}_{\text {Intf } m, k}$ increases. This fact is caused by the negative property of $a_{m}$.
Mathematically, the game $G$ can be expressed as:

$$
\begin{gathered}
\text { Find } P_{m, k}=\arg \max _{P_{m, k}} U_{m, k}\left(P_{m, k}, \mathbf{P}_{-m, k}\right) \\
\text { choose } P_{m}=\min _{k} P_{m, k}
\end{gathered}
$$

subject to:

$$
\left\{\begin{array}{l}
\sum_{m=1}^{M} P_{m, k}\left|h_{k, m}\right|^{2} \leq P_{T_{k}} \\
P_{\text {out }_{k}} \leq P_{\text {out }_{\text {max }}} \\
0 \leq P_{m, k} \leq P_{\text {max }}
\end{array}\right.
$$

Recall that $P_{m, k}$ denotes the strategy adopted by SU $m$ and $\mathbf{P}_{-m, k}=\left(P_{l, k}\right)_{l \neq m, l \in\{1, \ldots, M\}}$ denotes the strategy adopted by the other SUs with respect to each PU. The maximization problem is dependent on $a_{m}$ which is defined in the utility function as an adjustment parameter to the price function. For simulation results $a_{m}=-0.2$. It was chosen to stay with this value after different simulations to show its influence on the obtained results.

Our main contribution within this work is the QoS management of the CR system. The originality in the proposed method is that we guarantee a QoS to PU by maintaining the PU's outage probability unaffected in addition to a certain QoS to SUs and ensuring the continuity of service even when the spectrum sub-bands change from vacant to occupied. Thus by the outage probability control, if we have a vacant spectrum holes in the PU band, we set the outage probability $P_{\text {out }}=1$ to exploit the available spectrum band by SUs, and if we have occupied sub-bands, the outage probability is set to

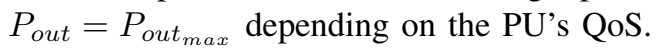

\section{NumERICAL RESUlTS}

For simulations, we consider a CRN with two femtocells. The primary system-like one is equipped with $K$ PUs and the secondary-like one with $M$ SUs attempting to communicate during a transmission, subject to mutual interference. A hexagonal cellular system functioning at $1.8 \mathrm{GHz}$ with a primary cell of radius $R=1000$ meters and a primary protection area of radius $R p=600$ meters is considered. Secondary transmitters may communicate with their respective receivers of distances $d<R p$ from the base station of the femtocell acting as a primary system. Channel gains are based on the COST-231 path loss model including log-normal shadowing with standard deviation of $10 \mathrm{~dB}$ [18], plus fast-fading assumed to be i.i.d. circularly symmetric with distribution $\mathrm{CN}(0 ; 1)$.

Fig. 2 and 3 present the results in terms of achieved capacity for each femtocell with our proposed scheme using inter cell handover based cooperative technique. The results show a dramatically improvement of the capacity for both cells. This is mainly due to the fact that the links between the users (from both femtocells) and the serving femtocell have better channel gains thanks to our proposed cooperative scheme. Fig. 4 shows the average transmitted power per user in both cells. It is clear that the proposed cooperative scheme improves the energy efficiency and reduce the power consumption by more than $5 \mathrm{dBm}$. 


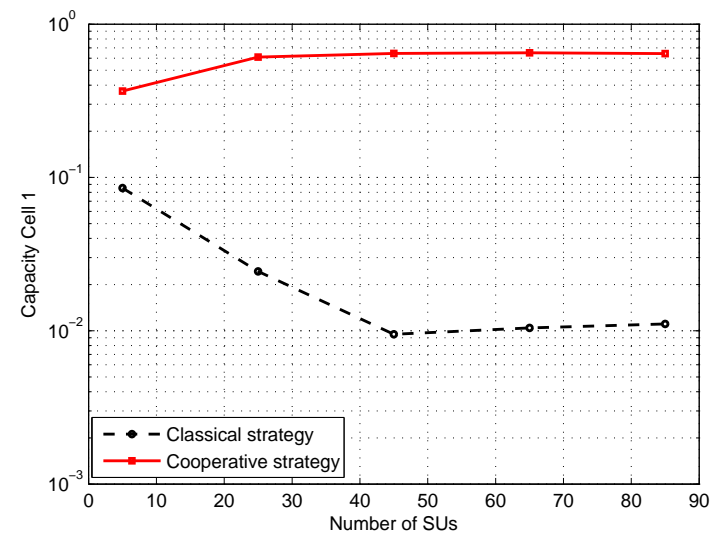

Fig. 2. Comparison of achieved capacity using inter cell handover based cooperative technique and classical technique for femtocell 1.

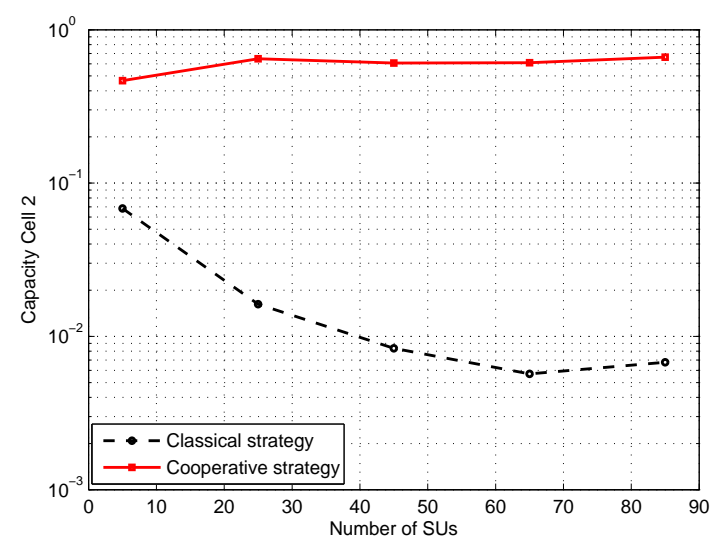

Fig. 3. Comparison of achieved capacity using inter cell handover based cooperative technique and classical technique for femtocell 2.

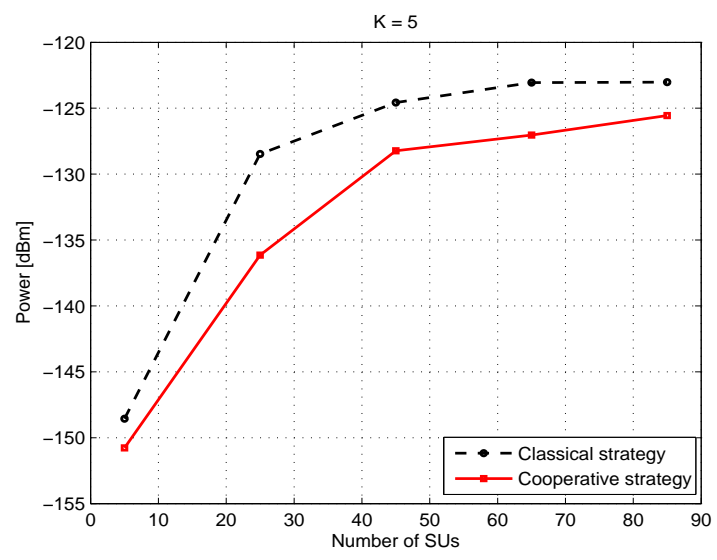

Fig. 4. Average transmitted power per user in both cells using inter cell handover based cooperative technique and classical technique.

\section{CONCLUSion}

In this paper, we explored a new cooperative scheme, in the context of cognitive femtocells, based on inter cell handover.
The results, carried in the context of an uplink scenario, show a dramatically system performance improvement when we take advantage from multi femtocells diversity and allow to users from competing femtocells to attach to the best serving cell. Similar results are expected for downlink scenario.

\section{ACKNOWLEDGEMENTS}

The research leading to these results has received funding from the European projects SACRA (spectrum and energy efficiency through multi-band cognitive radio) and ACROPOLIS (advanced coexistence technologies for radio optimisation in licensed and unlicensed spectrum), and was partially supported by the Hassan II Foundation for the Moroccans residing abroad.

\section{REFERENCES}

[1] K. Deshmukh, D. Patel, N. Gupta and S. Kumar, "Efficient Coding Mechanism for Low Power Consumption in Wireless Programmable Devices", International Journal of Soft Computing, vol 1, 2007.

[2] G. P. Perrucci, F.H.P. Fitzek, G. Sasso, W. Kellerer, J. Widmer; "On the Impact of 2G and 3G Network Usage for Mobile Phones Battery Life", Euroepan Wireless Conference, 2009.

[3] N. Balasubramanian, A. Balasubramanian and A. Venkataramani "Energy Consumption in Mobile Phones : A Measurment Study and Implications for Networks Applications", Proceedings of the 9th ACM SIGCOMM conference on Internet measurement conference(ICM), 2009.

[4] K. Lias, D.A.A. Mat, K. Kipli, A.S.W. Marzuki, "Human health Implication of $900 \mathrm{Mhz}$ and $1800 \mathrm{Mhz}$ mobile phones", IEEE 9th Malaysia International Conference on Communiction (MICC), 2009

[5] B. Zayen, A. Hayar and G. Noubir "Utility Pricing based Resource Allocation Strategy for Cognitive Radio Systems", ICMCS, Morocco 2011.

[6] J. Mitola, "Cognitive radio: An integrated agent architecture for software defined radio," Doctor of Technology, Royal Inst. Technol. (KTH), Stockholm, Sweden, 2000.

[7] S. Haykin, "Cognitive radio: Brain-empowered wireless communications", IEEE Journal on Selected Area in Communications, vol.23, no 2, pp. 201-220, Feb. 2005.

[8] B. Zayen, M. Haddad, A. Hayar and G. E. Øien, "Binary Power Allocation for Cognitive Radio Networks with Centralized and Distributed User Selection Strategies", Elsevier Physical Communication Journal, Vol. 1, No. 3, pp. 183-193, September 2008.

[9] B. Zayen, "Spectrum Sensing and Resource Allocation Strategies for Cognitive Radio", Doctor of Electronic and Communications, TELECOM ParisTech, Paris, France, November 2010.

[10] L.H. Ozarow, S. Shamai and A.D. Wyner, "Information theoretic considerations for cellular mobile radio," IEEE Trans. Veh. Technol., vol.43, no. 5, pp. 359-378, May 1994.

[11] S. G. Kiani, G. E. Øien, and D. Gesbert, "Maximizing multi-cell capacity using distributed power allocation and scheduling", in Proc. IEEE Wireless Communications and Networking Conference, Hong Kong, China, March 2007.

[12] J. M. Peha, "Approaches to spectrum sharing", IEEE Commun. Mag., vol. 43, no. 2, pp. 10-12, Feb. 2005.

[13] A. Jovicic and P. Viswanath, "Cognitive Radio: An InformationTheoretic Perspective", IEEE International Symposium on Information Theory, Seattle, USA, July 2006.

[14] D. Fudenberg and J. Tirole, "Game Theory", Cambridge, MIT Press, 1991.

[15] M. J. Osborne, “An introduction to game theory," Oxford University Press, August 2003.

[16] R. W. Rosenthal, "A class of games possessing pure-strategy Nash equilibria," International Journal of Game Theory 2, pp. 65-67. 1973.

[17] A. B. MacKenzie, L. Dasilva, and W. Tranter, "Game Theory for Wireless Engineers," Morgan and Claypool Publishers, 2006.

[18] Urban Transmission Loss Models for Mobile Radio in the 900 and 1800 MHz Bands, EURO-COST Std. 231, 1991. 